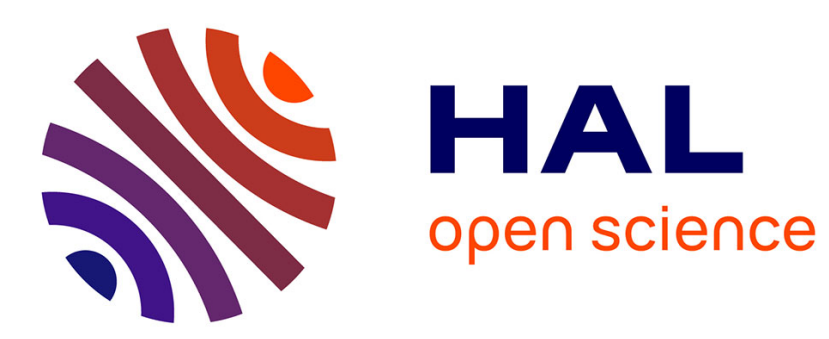

\title{
Edges, Transitions and Criticality
}

Suman Kumar Maji, Hussein Yahia

\section{To cite this version:}

Suman Kumar Maji, Hussein Yahia. Edges, Transitions and Criticality. Pattern Recognition, 2014, 10.1016/j.patcog.2013.12.013 . hal-00924137

\section{HAL Id: hal-00924137 \\ https://hal.inria.fr/hal-00924137}

Submitted on 6 Jan 2014

HAL is a multi-disciplinary open access archive for the deposit and dissemination of scientific research documents, whether they are published or not. The documents may come from teaching and research institutions in France or abroad, or from public or private research centers.
L'archive ouverte pluridisciplinaire HAL, est destinée au dépôt et à la diffusion de documents scientifiques de niveau recherche, publiés ou non, émanant des établissements d'enseignement et de recherche français ou étrangers, des laboratoires publics ou privés. 


\title{
Edges, Transitions and Criticality
}

\author{
Suman Kumar Maji* and Hussein M. Yahia \\ Geostat Team (Geometry and Statistics in Acquisition Data) of INRIA, \\ 200 rue de la Vieille Tour, 33405 Talence Cedex, FRANCE \\ Email: sumankumar.maji, hussein.yahia@inria.fr
}

\begin{abstract}
In this article, various notions of edges encountered in digital image processing are reviewed in terms of compact representation (or completion). We show that critical exponents defined in Statistical Physics lead to a much more coherent definition of edges, consistent across the scales in acquisitions of natural phenomena, such as high resolution natural images or turbulent acquisitions. Edges belong to the multiscale hierarchy of an underlying dynamics, they are understood from a statistical perspective well adapted to fit the case of natural images. Numerical computation methods for the evaluation of critical exponents in the non-ergodic case are recalled, which apply for the vast majority of natural images. We study the framework of reconstructible systems in a microcanonical formulation, show how it redefines edge completion, and how it can be used to evaluate and assess quantitatively the adequation of edges as candidates for compact representations. We study with particular attention the case of turbulent data, in which edges in the classical sense are particularly challenged. Tests are conducted and evaluated on a standard database for natural images. We test the newly introduced compact representation as an ideal candidate for evaluating turbulent cascading properties of complex images, and we show better reconstruction performance than the classical tested methods.
\end{abstract}

Keywords: Edge detection, complex systems, statistical physics, critical exponents, compact representation, nonlinear signal processing, multifractals, wavelets, multiresolution analysis, turbulence, scale space.

${ }^{*}$ Corresponding author. Tel.: (+33)(0)5 245740 49, Fax : (+33)(0)5 24574124 . 


\section{Introduction}

As algorithms dedicated to the computation of edges in digital images emerged $[1,3,4,7,5,6,9,10,11,12,13,14,36,15]$, Torre and Poggio [16], while observing that most methods rely on the ill-posed problem of differentiating digital images, proposed a general qualitative description of edges: they noted that edges are naturally associated to the concepts of compact representation (they call it completion), i.e. edges encode most information of an image [57]. Similarly, other authors note that edges represent an image's independent features [20]. In [16] the authors focus on edge detection as the process of computing derivatives, and, while attempting to do so in a well-posed form, they are led naturally to the problem of prefiltering the image by a (e.g. Gaussian) kernel, which transforms the input signal into a differentiable mapping in the continuous domain, hence allowing the characterization of edges by differential operators. An instance of this formalism is the zero-crossing of second-order derivatives, as in $[10,17,11,14,52,55,56]$, or [44] to cite few, including a recent nonlinear derivative approach (called NLFS) [53]. This formal setting allowed the development of edge characteristics in the framework of differential geometry, a perspective that has become pervasive in image processing $[18,34]$. The multiscale nature of edges was recognized very early and it was noted that tracing edge properties across scales would gain insight into the physical process behind image formation. Neurophysics was demonstrating that, in the optical pathway, spatial filters of different sizes operate at the same location [19]. This is related to the processing of information in the early visual system [24], where cells tend to take advantage of the statistical regularities of the input signal in order to get compact representations out of redundancy [25, 26, 27].

The convolution of the input image signal by a Gaussian kernel introduces a scale parameter (the standard deviation of the Gaussian kernel) corresponding to a simple linear scale-space associated to the heat equation. This is often used as an argument for advocating multiscale properties of Gaussian prefiltering [16, 29, 32, 33, 37]. In general, however, the multiscale properties of complex systems do not comply with such an extreme simplification [28]. The advent of scale-space theory in Computer Vision allowed more complex multiscale representations corresponding, among others, to anisotropic diffusion schemes [30, 35, 46, 54], which can incorporate probabilistic models of both sensor noise and operators' responses (to better estimate the gradient's magnitude threshold in case of noise [47]). However the simple example 
of an image corresponding to the acquisition of a turbulent fluid, like, for instance, a remotely sensed acquisition over the oceans, contains coherent structures associated to the cascading properties of intensive variables in Fully Developped Turbulence (FDT) [42]. It has an associated multiscale hierarchy consisting of sets having a multifractal nature [31] and, as such, cannot be contemplated within a differentiable scale-space framework. Incidentally note that in [49] authors write that an appropriate spatial scale depends upon the local structure of the edge, and thus varies unpredictably over the image.

In a seminal paper, Mallat and Zhong [38] relate multiscale Canny edge detection to the local maxima of a wavelet transform and study the completion of multiscale edges associated to the maxima of wavelet coefficients (multiscale edge detection $[38,58]$ ); for that purpose they introduce a reconstruction algorithm of a signal from its edges. The quality of the reconstruction is quantitatively evaluated by the SNR of the original and reconstructed signals, hence providing an accurate evaluation of the quality of edge pixels determined by their method; this evaluation is different than the previous criteria used in computing edge detection performance $[18,43]$. Local maxima of wavelet coefficients are also used by other authors to form the basis of the Wavelet Transform Modulus Maxima (WTMM) methodology [40]. Edges can also be understood as alignment of Fourier or wavelet phases across scales $[21,22,23]$.

In this paper, we show that recent developments around the notion of transition in nonlinear physics, along with enhanced computational methods of its quantitative parameters (most notably singularity exponents) [42], lead to a notion of edge that provides better results over all the previous declined versions encountered in image processing w.r.t. edge completion. Our results strongly advocate for a definition of edge based on nonlinear operators while we prove along the way, and incidentally, that previous nonlinear approach [53] also works better than the classical ones w.r.t. to reconstruction of an image from its edge data. This can be done by referring to the earlyaddressed pertinent notion of compact representation (completion). When neurophysics and the study of biological vision in mammals state that edges encode most information in an image signal, this must have the consequence of being able to reconstruct accurately an image from the compact representation of its edge pixels ([58], p. 194). Statistically, image acquisitions correspond to processes out of the equilibrium state, so that, from a theoretical point of view, transitions associated to scale-space formulations reviewed 
above cannot be correct. We show that the new notion of edge outperforms the most well-known previous ones and that it is naturally robust to noise. We give specific attention to the case of turbulent images, whose edges are not well defined in the classical context of edge detection, and we show that in this context the new notions introduced in this article work much better than the previous ones.

Unpredictability of edges, the concept of singularity exponents and the framework of reconstructible systems are introduced in section 2. Edge consistency across the scales is addressed in section 3. Quantitative results are shown and discussed in section 4 and section 5, where in subsection 4.4 the case of turbulent images is specifically addressed. Conclusion (section 6) follows.

\section{Edges, unpredictability and reconstructibility revisited using the microcanonical multiscale formalism (MMF)}

In this section we show how the microcanonical multiscale formalism (MMF [71]) can be applied to edge detection and image reconstruction. We will show that ideas borrowed from Statistical Physics about criticality and exponents, when evaluated in a microcanonical formulation, are associated to a computable notion of transition, intimately related to predictability in complex systems [59]. This in turn gives rise to a notion of edge whose quantitative performance evaluation can be tested through the framework of reconstructible systems.

\subsection{Local Predictability Exponents}

Classically, edges are related to sharp variations of the image gradient. The main idea worked out in this article is to delve deeper into developing the notion of "sharp variation", and relate it to the more general notion of "transition" defined in Statistical Physics for intensive physical variables. For that matter, a scalar image $I$ defined over a compact subset of $\mathbb{R}^{2}$ is identified with an intensive physical variable (such an identification corresponds exactly to the physics of the acquisition for images of natural phenomena such as in infrared remote sensing imagery etc.). In nonlinear physics, the relation between the transitions of an intensive variable and criticality is well known, explained, and quantitatively formalized through the notion of critical exponent [62]. We recall that definition here. We say that image $I$ has 
a critical exponent $h(\vec{x})$ at point $\vec{x}$, if for at least one multiscale functional $\mathbb{T}_{r}{ }^{1}$, dependent on scale $r$, the following equation holds:

$$
\mathbb{T}_{r} I(\vec{x})=\alpha(\vec{x}) r^{h(\vec{x})}+o\left(r^{h(\vec{x})}\right)(\vec{r} \rightarrow 0)
$$

where $\alpha(\vec{x})$ is a signal-dependent amplitude prefactor. An effective choice for the functional $\mathbb{T}_{r}$ leads to a measure given by the total variation of the image gradient [71], and is defined as:

$$
\mu\left(\mathcal{B}_{r}(\vec{x})\right)=\int_{\mathcal{B}_{r}(\vec{x})}\|\nabla I\|(\vec{x}) \mathrm{d}(\vec{x})
$$

where $\mathcal{B}_{r}(\vec{x})$ is a ball of radius $r$ centered at point $(\vec{x})$ of the signal domain. The wavelet transform of the measure $\mu$ then allows us to infer a more computable version of the singularity exponents (less prone to noise variation) [72], such that:

$$
\mathcal{T}_{\Psi} \mu(\vec{x})=\alpha_{\Psi}(\vec{x}) r^{h(\vec{x})}+o\left(r^{h(\vec{x})}\right)(r \rightarrow 0)
$$

where $\Psi$ is an associated wavelet.

The central problem is to compute at high numerical precision the value of $h(\vec{x})$ at point $\vec{x}$ : bad approximations of singularity exponents lead to poor reconstructions. We will address the problem of robust computation of singularity exponents in section 2.2. For the moment we note that equation (1) is a pointwise and localized version of the definition used in introducing singularity spectrum $[40,70]$ : we don't make use of statistical averages and grand ensembles, but seek to evaluate $h(\vec{x})$ at point $\vec{x}$ (which means we cannot rely on stationarity hypothesis). We denote $\mathcal{F}_{h}$ the component in the image's domain associated to singularity exponent value $h$ as:

$$
\mathcal{F}_{h}=\{\vec{x}: h(\vec{x})=h\}
$$

This family of sets is naturally associated to the multiscale hierarchy in an image [71]. In the case of natural images of the physical world, it is expected that the values of $h(\vec{x})$ are bounded by below, so that the Most Singular Manifold or MSM can be defined as:

$$
\mathcal{F}_{\infty}=\left\{\vec{x}: h(\vec{x})=h_{\infty}=\operatorname{Min}(h(\vec{x}))\right\}
$$

\footnotetext{
${ }^{1}$ Typical examples of $\mathbb{T}_{r}$ can be wavelet transforms at scale $r$ or some differential operators applied to the signal.
} 
noting that, in digital signals, the value $h_{\infty}$ is thresholded and must correspond to a (small) tolerance interval. The MSM comprises the set of points in an image with the sharpest transitions, corresponding to most unpredictable points. Consequently, if this set is computed properly, it must maximize the statistical information content in the image, so that we should be able to reconstruct the signal from its restriction to this set: this is the framework of reconstructible systems that will be our fundamental tool in evaluating the quality of a compact representation for a set of edges.

Criticality, and the associated critical exponents are key notions in Statistical Physics to understand phase transitions, which are prototypes of scale invariant phenomena. In high order transitions, thermodynamical variables as well as time and spatial correlation functions display a power-law behaviour, where the characteristics of the underlying system are determined completely, at the macroscopic scale, by the value of the exponent [62]. Critical exponents are universal: systems having the sames values of critical exponents belong to a same universality class and their statistical macroscopic multiscale characteristics are similar, independantly of the causalities in the extended phase space. This is used in recent advances in complex systems and complex network methods, where fingerprints of universality classes are checked from singularity spectra computed from critical exponents [69], or the statistics in natural images are found identical to those of complex systems [24]. The distribution of critical exponents in a system determines its multiscale properties which are accessible statistically. In particular, the classical notion of gradient, which serves as the basic ingredient in most edge detection algorithm is not clearly defined in such context and one must find more accurate statistical description of transitions across the scales.

\subsection{Computation of the singularity exponents}

Following equation $(1), h(\vec{x})$ can be obtained by a linear regression of $\log \left(\mathbb{T}_{r} I(\vec{x})\right)$ vs. $\log (r)$ :

$$
h(\vec{x})=\lim _{r \rightarrow 0} \frac{\log \left(\mathbb{T}_{r} I(\vec{x}) / \alpha(\vec{x})\right)}{\log r}
$$

A very fast but crude version of computing $h(\vec{x})$ is known as the Gradient histogram method [41], which takes into account the multiscale measure defined in equation (2), at a minimum resolution $r_{0}$. The scale $r_{0}$ is chosen such that the whole image corresponds to size 1 ; in other words, if the image 
is an array of discretize values of size $m \times n$, one chooses $r_{0}=1 / \sqrt{m \times n}$. Approximating $\alpha(\vec{x})$ as the average of the norm of the gradients, an estimate of $h(\vec{x})$ is obtained as:

$$
\tilde{h}(\vec{x}) \equiv \frac{\log (\|\nabla I\|(\vec{x}) /\langle\|\nabla I\|\rangle)}{\log r_{0}}
$$

The method however fails to produce satisfactory results for natural images and is highly sensitive to noise [41]. A better solution would consist in perfoming the regression on wavelet projection of measures [71], i.e., a logarithmic regression applied to equation (3). However, a wavelet projection of the measurement at various scales is costly in computation time and only serves to enhance the resolution of less singular structures at the cost of coarsening the most singular ones [41]. Since the objective is to recover the most singular structures, a better optimized and cost-effective way is to use a point estimation of the singularity exponents. From the perspective of reconstructible systems, good evaluation algorithms come from the observation that the set of most unpredictable points $\mathcal{F}_{\infty}$ (see equation (5)) that provides a perfect reconstruction is such that [72]:

$$
\operatorname{div}\left(\left.\nabla I\right|_{\mathcal{F}_{c}}\right)=0
$$

where $\mathcal{F}_{c}$ is the complementary set of $\mathcal{F}_{\infty}$. As a consequence, singularity exponents can be called Local Predictability Exponents, they encode predictability information [59], like Lyapunov exponents, and are better evaluated in digital signals by one of the following approximation:

$$
h(\vec{x})=\frac{\log \mathcal{T}_{\Psi} \mu\left(\vec{x}, r_{0}\right) /\left\langle\mathcal{T}_{\Psi} \mu\left(\cdot, r_{0}\right)\right\rangle}{\log r_{0}}+o\left(\frac{1}{\log r_{0}}\right)
$$

where $\left\langle\mathcal{T}_{\Psi} \mu\left(\cdot, r_{0}\right)\right\rangle$ is the average value of the wavelet projection over the whole signal, and $r_{0}$ is chosen to diminish the relative amplitude of the correction term $o\left(\frac{1}{\log r_{0}}\right)$. When the values of $h(\vec{x})$ are computed for all points $\vec{x}$ in the image's domain, they can be sorted and the typical threshold 0 is naturally chosen to define the standard reconstruction set in the MSM method. Such a set often provides a very good reconstruction. Consequently the basic edge set computed automatically in the MSM edge detection method, that yields a good reconstruction, and as such can define the support of a complete compact representation, is defined by:

$$
\mathcal{E}_{\mathrm{MSM}}=\bigcup_{h_{\infty} \leq h \leq 0} \mathcal{F}_{h}
$$


Table 1: Images used for our experiments. Row 1 (from left to right): Harrington weave (SIPI image database), Hibiscus (SIPI image database), car (CMU image database), Lena (SIPI image database), imk01310 (van Hateren image database), imk03324 (van Hateren image database), imk04050 (van Hateren image database). Row 2: visualization of the singularity exponents. Row 3: compact representation of MSM points corresponding to $30 \%$ pixel density.

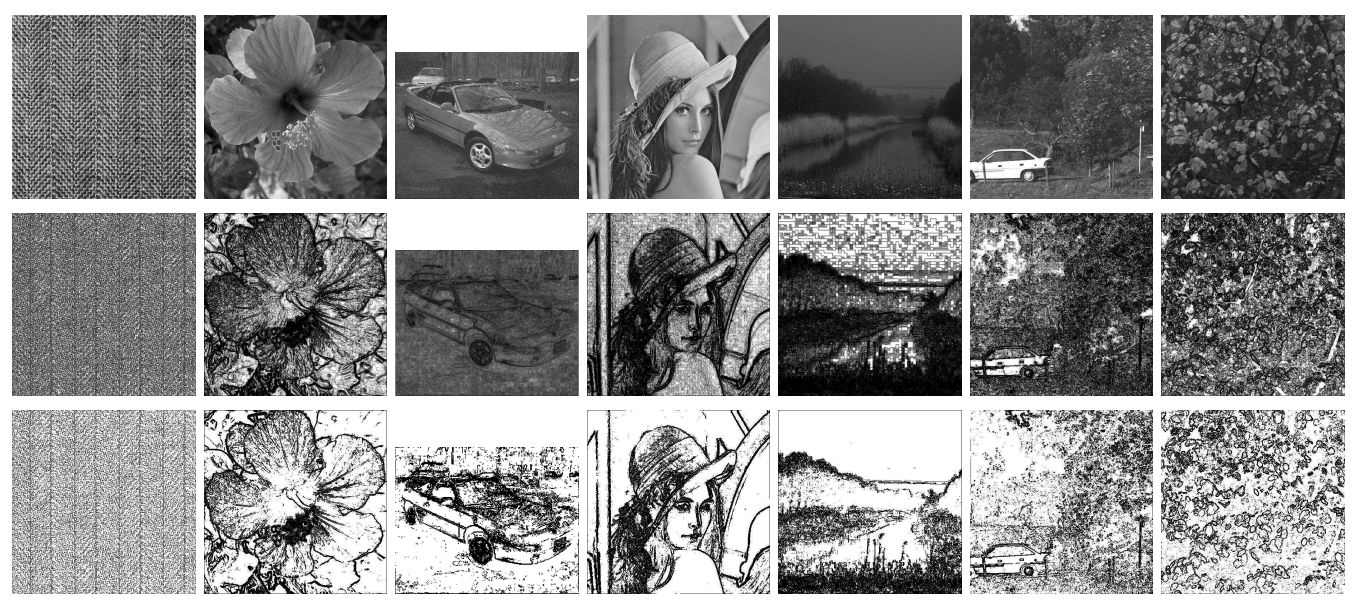

In general, $\mathcal{E}_{\mathrm{MSM}}$, as well as $\mathcal{F}_{\infty}$ are multifractal, so that a notion of unitary orthogonal field to these sets can be defined only statistically across the scales. A normal unitary field to $\mathcal{E}_{\mathrm{MSM}}$ is computed in Fourier space by prefiltering it with nonlinear kernel $\widehat{W}(\vec{k})=\frac{1}{\|\vec{k}\|}$ corresponding to the convolution with wavelets from the family $\Psi^{\beta}(\vec{x})=1 /\left(1+|\vec{x}|^{2}\right)^{\beta}$, for $\beta=1,2,3,4$, and averaging the resulting coefficients. Images used for our experiments and their corresponding MSM points are shown in Table 1. For the next part of the paper, we will call our edge detection algorithm corresponding to equations (9) and (10) as $\mathbf{I}_{\infty}$.

\subsection{Reconstructible Systems}

In this section, we are led to find mathematically a functional $\mathcal{G}: L_{\mathbb{C}}^{1}\left(\mathbb{R}^{2}\right) \rightarrow$ $L_{\mathbb{C}}^{1}\left(\mathbb{R}^{2}\right)$ which permits the reconstruction of the signal's gradient measure from its restriction to the MSM $\mathcal{F}_{\infty}$. The choice of the functional is made under the conditions of verifying the properties of being deterministic, linear, translationally-invariant, isotropic and yield correct power spectrum of natural images [63]. The set $\mathcal{F}_{\infty}$ being equal to the set of most unpredictable points, the functional $\mathcal{G}$ is reconstructing the whole gradient $\nabla I(\vec{x})$ from 
the knowledge of $\left.\nabla I\right|_{\mathcal{F}_{\infty}}$ (i,e, the gradient restricted to the set of most unpredictable points). By using the Riesz integral representation theorem for linear continuous functionals and using translational invariance one gets

$$
\nabla I(\vec{x})=\int_{\mathcal{F}_{\infty}} \nabla I(\vec{y}) G(\vec{x}-\vec{y}) \mathrm{d} \vec{y}
$$

where $\int_{\mathcal{F}_{\infty}} \mathrm{d} \vec{y}$ means integration over the MSM and we represent the linear operator $\mathcal{G}$ by means of its density matrix, denoted by $G(\vec{x}, \vec{y})$, as given by the theory of linear representation.

The above equation can rewritten in the form of a distributional field which contains all the functional (the values of the gradient $\nabla I$ ) and geometrical (the MSM) information which is specific to the signal to be reconstructed. The essential gradient of the signal $\nabla_{\infty} I(\vec{x})$ is defined as the following distribution:

$$
\nabla_{\infty} I(\vec{x})=\nabla I(\vec{x}) \delta_{\mathcal{F}_{\infty}}(\vec{x})
$$

where $\delta_{\mathcal{F}_{\infty}}(\vec{x})$ is the density of the gradient measure restricted to the MSM. Equation (12) results in a standard convolution as the integration is now performed over all the space and not restricted to the MSM. A practical expression of the reconstruction formula is given by [63]:

$$
I(\vec{x})=\int\left\langle\vec{g}(\vec{x}-\vec{y}) \mid \nabla_{\infty} I(\vec{y})\right\rangle \mathrm{d} \vec{y}=\vec{g} * \nabla_{\infty} I(\vec{x})
$$

for a vector $\vec{g}$, that is to say the form of a diffusion kernel, but integrated over the multifractal set of most unpredictable points $\mathcal{F}_{\infty}$, and is expressed in the Fourier domain as:

$$
\hat{I}(\vec{\omega})=\left\langle\hat{\vec{g}}(\vec{\omega}) \mid \widehat{\nabla_{\infty} I}(\vec{\omega})\right\rangle
$$

where $\langle\cdot \mid \cdot\rangle$ denoting the standard Hermitian product on $\mathbb{C}^{2}$ and $\widehat{ }$ represents the Fourier transform.

\subsection{Determination of the kernel}

To derive an exact formulation for the reconstruction kernel $\hat{\vec{g}}$, we use an alternate approach to [63] and follow the derivation presented in [64] in the case of differentiable fields. For that matter, given a predefined smooth vector 
field $\vec{v}$, one can seek an image $I(\vec{x})$ such that its gradient $\nabla I(\vec{x})$ minimizes the $L^{2}$ distance with $\vec{v}$ as

$$
\underset{\mathrm{I}}{\operatorname{argmin}} \iint\|\nabla I(\vec{x})-\vec{v}(\vec{x})\|_{2}^{2} \mathrm{~d} \vec{x}
$$

Following the derivation explained in [64] we get a version of the reconstruction kernel $\hat{\vec{g}}$ in the smooth case as follows. We take the Euler-Lagrange variational formulation of equation (15) and obtain

$$
\operatorname{div}(\nabla I)(\vec{x})=\operatorname{div}(\vec{v})(\vec{x})
$$

Taking the Fourier transform of equation (16), one gets

$$
\hat{I}(\vec{\omega})=-i \frac{\omega_{x} \hat{v}_{x}(\vec{\omega})+\omega_{y} \hat{v}_{y}(\vec{\omega})}{\omega_{x}^{2}+\omega_{y}^{2}}
$$

where the vector field $\vec{v}(\vec{x})$, after Fourier transformation gives rise to a complex vector field $\hat{\vec{v}}=\left(\hat{v}_{x}(\vec{\omega}), \hat{v}_{y}(\vec{\omega})\right)$. Considering equation (14), this suggests the kernel as

$$
\hat{\vec{g}}(\vec{\omega})=\frac{\vec{\omega}}{i\|\vec{\omega}\|^{2}}
$$

and consequently the following expression of the reconstruction formula in the Fourier domain:

$$
\hat{I}(\vec{\omega})=\frac{\left\langle\vec{\omega} \mid \widehat{\nabla_{\infty} I}(\vec{\omega})\right\rangle}{i\|\vec{\omega}\|^{2}}
$$

Fourier inversion of this formula gives the reconstruction of the image from the restriction of the gradient field to the MSM.

\section{Edge consistency across the scales}

Edges are primary features naturally associated to scale invariant properties of natural images, specifically in the case of turbulent signals where symmetry is restored only in statistical sense [24, 31]. As a consequence, the algorithms used in computing edge features should be consistent across the scales: if one has different acquisitions of a same phenomenon at different scales, the resulting edge pixels computed by these algorithms must produce matching edge pixels accross the scales. To check this, we set up an experiment where a same signal at different resolution is generated and 
their outputs produced by some classical edge detection algorithms are evaluated. To generate the different resolutions, we use two methods. The first one consists in computing a multiscale version of the signal by using a standard Haar discrete wavelet transform [58]. The second one consists in using the well-known linear scale-sace representation developed by Lindeberg et al $[60,61]$. A linear scale-space family $L(., t)$ associated to an original signal $f$ is obtained by convolving $f$ with a Gaussian kernel $g$ such that:

$$
L(., t)=g(., t) * f
$$

where the Gaussian kernel is given by

$$
g(x, t)=\frac{1}{2 \pi t} e^{-\left(x^{2}+y^{2}\right) / 2 t}
$$

and $t>0$ is the scale parameter.

We take two images: the clock image from SIPI image database [66], which is a standard rigid object, and an excerpt of a remotely sensed image of sea surface temperature (SST) acquired from space. The SST image corresponds to acquisition of a turbulent phenomenon, for which the existence of a multiscale hierarchy comes from the turbulence associated to Navier-Stokes equations [31]. The results for the SST image are shown in Table 2. In this table are diplayed (left column) the SST image at various resolutions using the Haar discrete wavelet transform. Columns 2, 3 and 4 show the edge pixels produced by three different algorithms: the algorithm called $\mathbf{I}_{\infty}$, which is the proposed edge detection algorithm (explained in section 2.2), classical Canny edge detector (column 3) and the Mallat-Zhong edge detection technique [38], also known as multiscale Canny edge detection (which is also related to the WTMM method). It is clear from these results that Canny edge pixels are not consistent across the scales: first the boundary of the main coherent structure (the temperature front depicted by the boundary between the dark and light area) is not properly described by Canny edge pixels across the scales. Second, it is difficult to match the corresponding edge pixels across the scales. This can, however, be related to the fact that Canny edge detector encodes all edges regardless of scale, and that is why we also use multiscale Canny in our comparison. Considering the multiscale Canny edge pixels, they behave more consistently compared to Canny edge pixels, but they are still outperformed by $\mathbf{I}_{\infty}$, the latter being specifically designed to retain consistency across the scales. This is confirmed by the results displayed in columns 5, 6, 7 of Table 2, which shows the reconstruction 
Table 2: Inconsistent behaviour of edges along the scales. Image used is an excerpt from the sea surface temperature (SST) image (MODIS data) of the Agulhas current below the coast of South Africa. Different resolutions of the SST image are formed by taking the approximation coefficients resulting from a standard Haar discrete wavelet transform. The edge pixels are marked red or blue according to the sign of the scalar product between the normal to the set of edge and the image gradient at that point (see section 2.2 after equation (10)). We show the results of the following edge detection algorithms: $\mathbf{I}_{\infty}$ (proposed edge detection algorithm, explained in section 2.2), Canny and multiscale Canny (mC) edge detection [38]. D corresponds to the pixel density of the respective edges. For edges computed using Canny edge detector, $\alpha$ specifies the lower sensitivity threshold and $\sigma$ is the standard deviation of the Gaussian filter. R-I $\mathbf{I}_{\infty}, \mathrm{R}$-Canny and $\mathrm{R}-\mathrm{mC}$ are the reconstructions, with equation (19), from their respective edges.
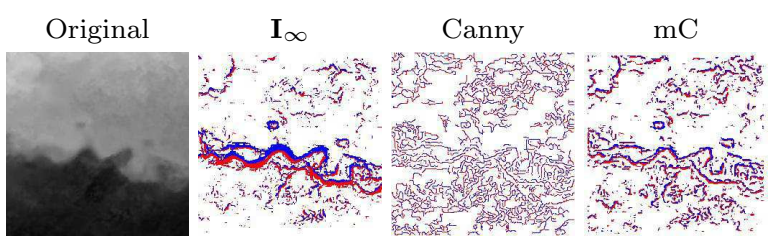

$256 \times 256$

$\mathrm{D}=16.24 \%$

pixels
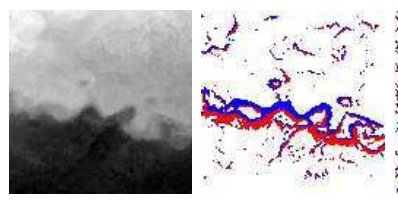

$\mathrm{D}=16.24 \%$

$\alpha=0.1, \sigma=0.03$

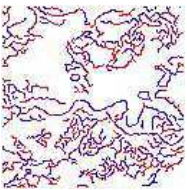

$\mathrm{D}=17.45 \%$

$\mathrm{D}=17.45 \%$
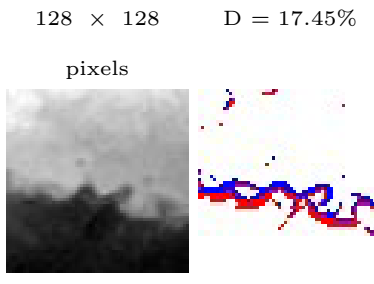

$\alpha=0.13, \sigma=0.03$

$\mathrm{D}=16.89 \%$

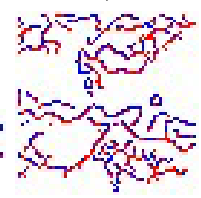

$\mathrm{D}=16.89 \%$

$\alpha=0.15, \sigma=0.03$
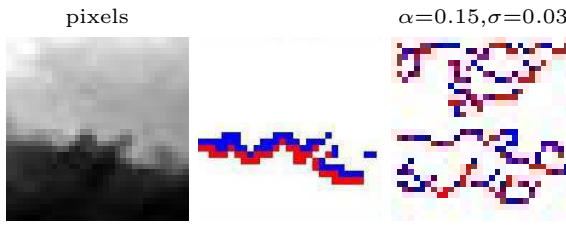

$\mathrm{D}=18.55 \%$

$\mathrm{D}=18.55 \%$

$\alpha=0.16, \sigma=0.03$
$\mathrm{D}=16.98 \%$

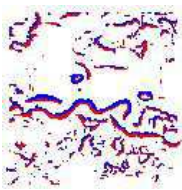

$\mathrm{R}-\mathbf{I}_{\infty}$

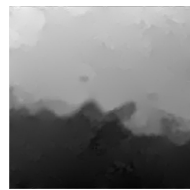

$\mathrm{psnr}=23.46 \mathrm{~dB}$
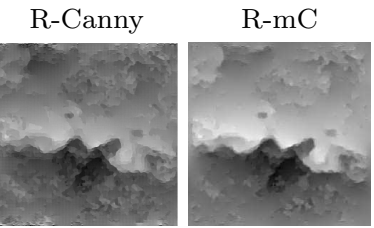

$\mathrm{psnr}=4.18 \mathrm{~dB}$

$\mathrm{psnr}=6.76 \mathrm{~dB}$
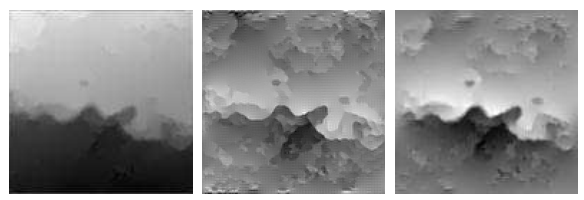

$\mathrm{psnr}=22.31 \mathrm{~dB}$

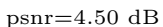

$\mathrm{psnr}=6.49 \mathrm{~dB}$

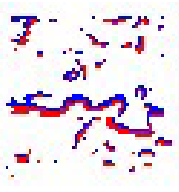

$\mathrm{D}=17.91 \%$
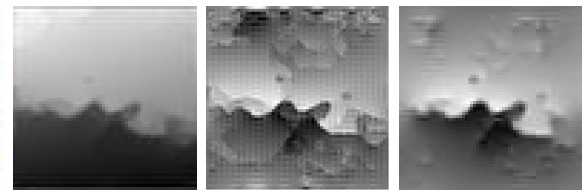

psnr $=20.65 \mathrm{~dB}$

$\mathrm{psnr}=3.77 \mathrm{~dB}$

psnr $=6.69 \mathrm{~dB}$

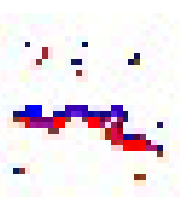

$\mathrm{D}=19.04 \%$
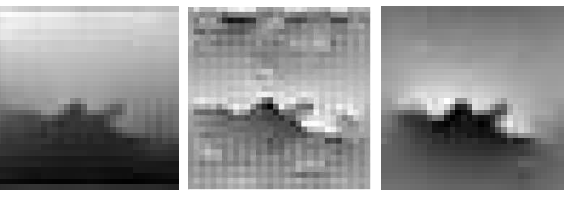

$\mathrm{psnr}=17.81 \mathrm{~dB}$

psnr $=3.52 \mathrm{~dB}$

$\mathrm{psnr}=6.30 \mathrm{~dB}$ 
Table 3: Inconsistent behaviour of edges along the scales. Image used is the clock image from SIPI image database [66]. Different resolutions of the clock image are formed by taking the approximation coefficients of Haar discrete wavelet transform.

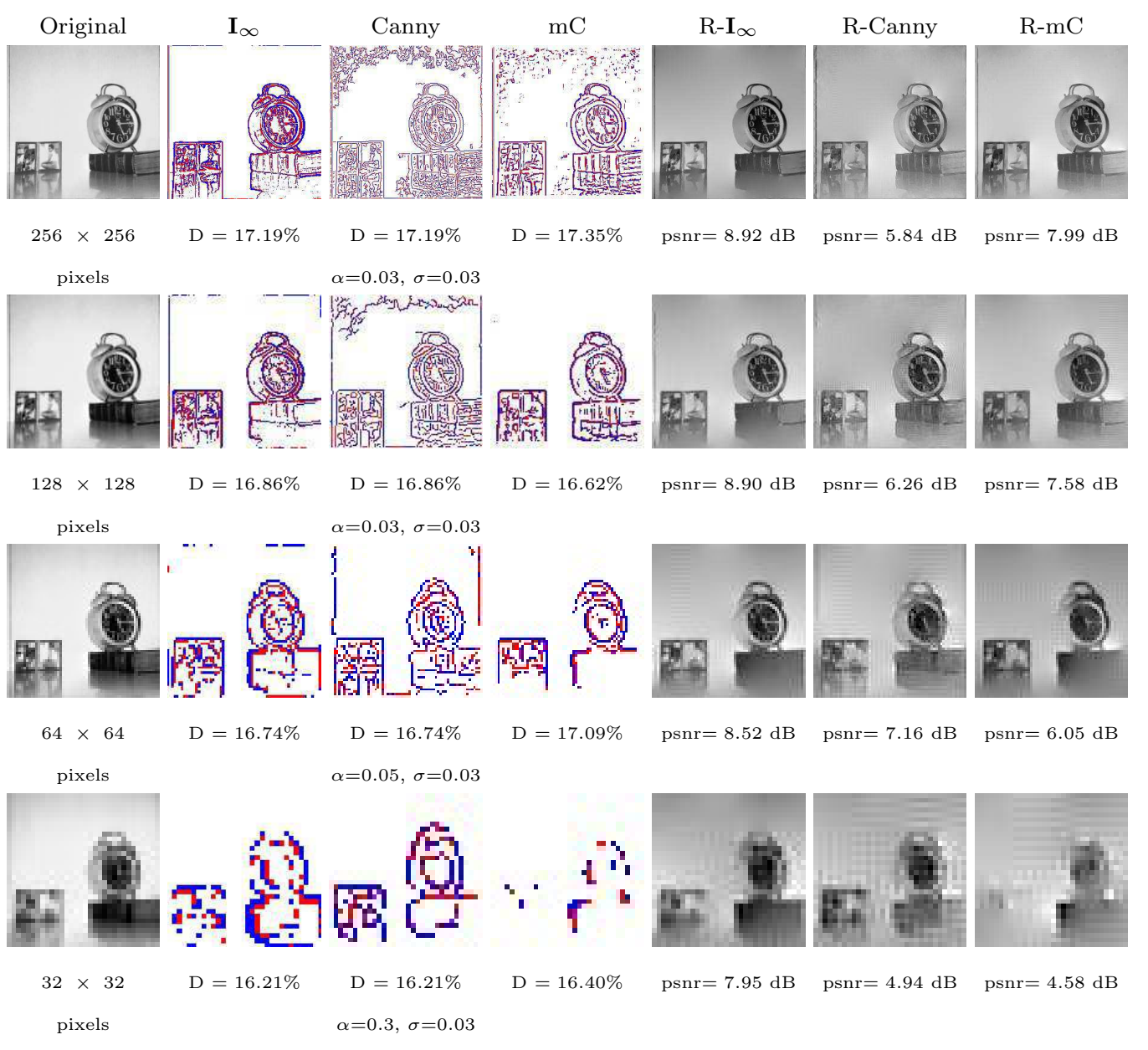

of the whole signal from its gradient measure restricted to the edge pixels. As explained later in this article, this is a crucial test, as edges are supposed to encode in the most efficient way the information content in the signal. The precise reconstruction process shown in this table is fully explained in section 2.3. Note that in Table 2 , edge pixels produced by $\mathbf{I}_{\infty}$ algorithm encode in a particularly efficient way the main boundary of the temperature front across the scales. The same is reproduced in Table 3 on the clock image, 
Table 4: Evaluation of edge consistency across scales using Lindeberg [60] scale space representation. Row 1 (from left to right): different resolutions of the clock image obtained by changing the scale parameter $t$ (explained in section 3). Row 2: compact representation of $\mathbf{I}_{\infty}$ points. Row 3: edges computed using Lindeberg edge detector.
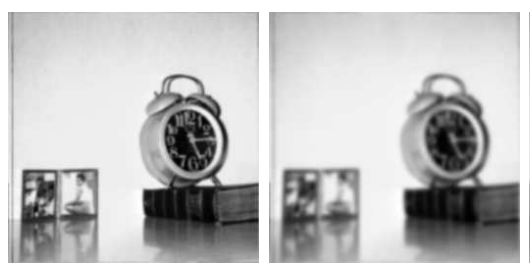

$\mathrm{t}=1.0$

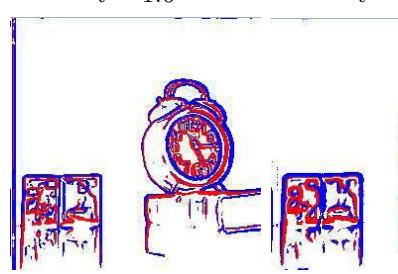

$\mathrm{t}=16.0$
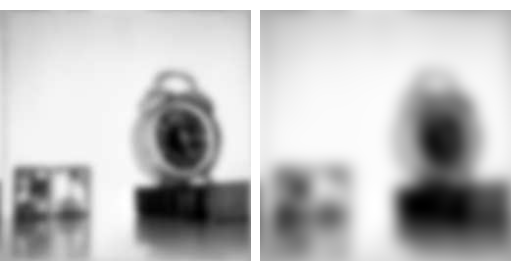

$\mathrm{t}=64.0$
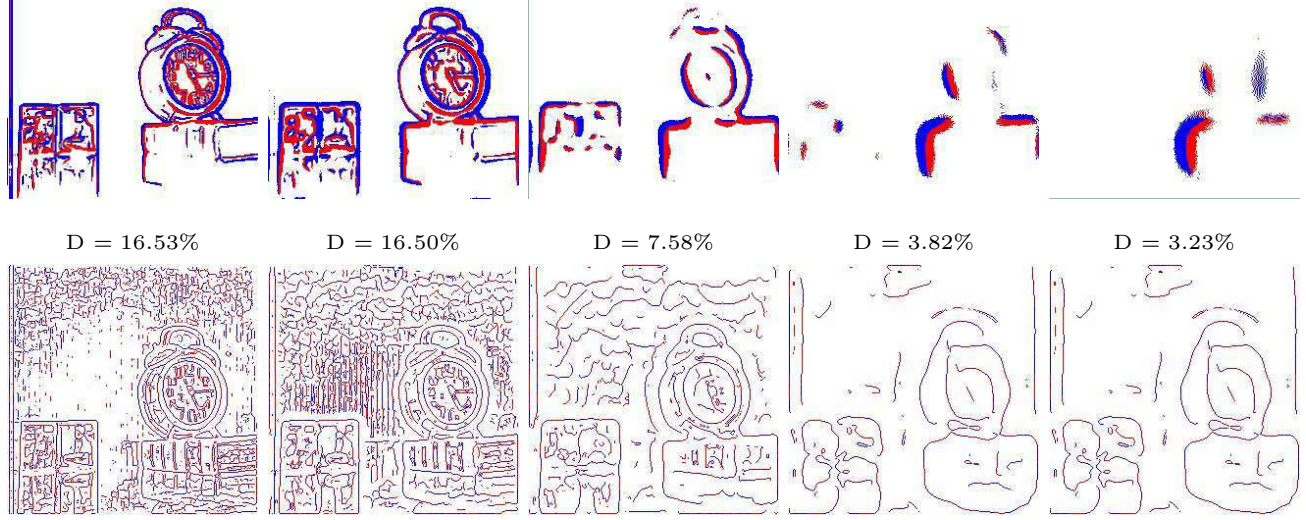

$\mathrm{D}=16.50 \%$

$\mathrm{D}=7.58 \%$

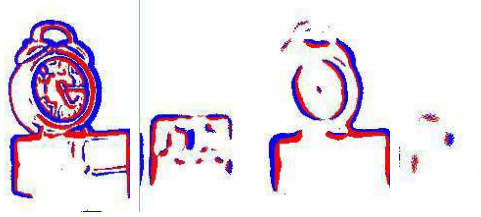

$\mathrm{D}=3.82 \%$
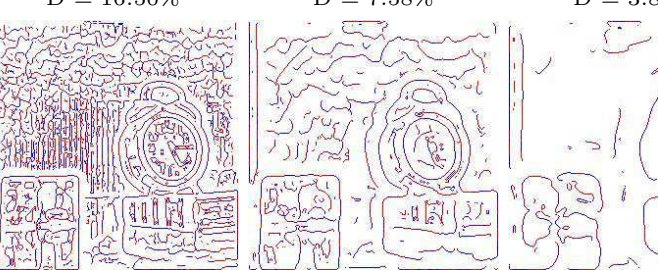

$\mathrm{D}=16.50 \%$

$\mathrm{D}=16.53 \%$

$\mathrm{D}=7.58 \%$

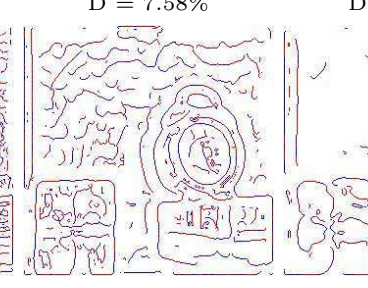

$\mathrm{D}=3.82 \%$

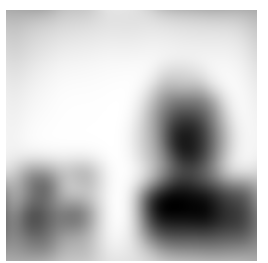

$\mathrm{t}=256.0$

with similar results. In Table 4 and Table 5, we make a similar comparison between the $\mathbf{I}_{\infty}$ edges and the edges produced by the multiscale edge detection of Lindeberg [60]. Here again, we see that in both cases, consistency across the scales is better achieved by $\mathbf{I}_{\infty}$ algorithm. In Table 5 , the density of the $\mathbf{I}_{\infty}$ edges are kept around $16 \%$ precisely; but because Lindeberg edges uses a zero-crossing of image intensity further filtered by a third order directional derivative, the number of candidate edge pixels is automatically reduced with increased blurring, resulting in a limited density across the scales. From these experiments we see that, specially in the case of acquired turbulent phenomena, some classical edge detection algorithms, even those based on linear scale-space theory, do not behave consistently across the scales. 
Table 5: Evaluation of edge consistency across scales using Lindeberg scale space representation. Row 1 (from left to right): different resolutions of the SST image obtained by changing the scale parameter $t$. Row 2: compact representation of $\mathbf{I}_{\infty}$ points (pixel density fixed at 16\%). Row 3: edges computed using Lindeberg edge detector.
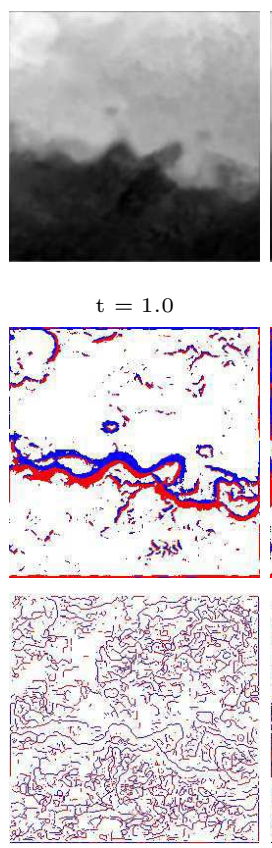

$\mathrm{D}=16.53 \%$

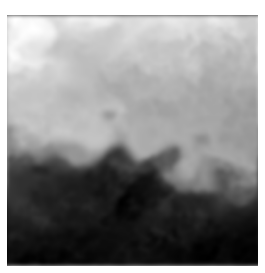

$\mathrm{t}=4.0$

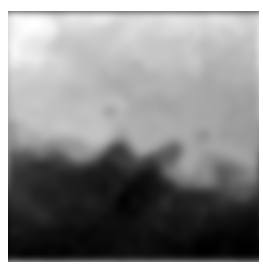

$\mathrm{t}=16.0$

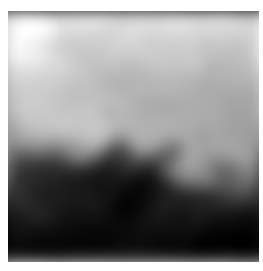

$\mathrm{t}=64.0$

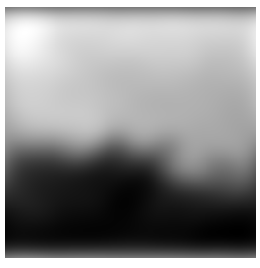

$\mathrm{t}=256.0$

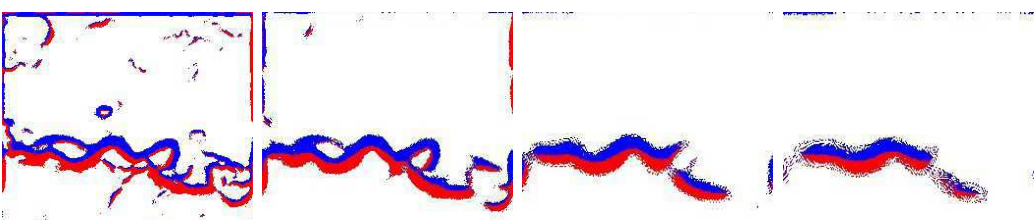

$*$
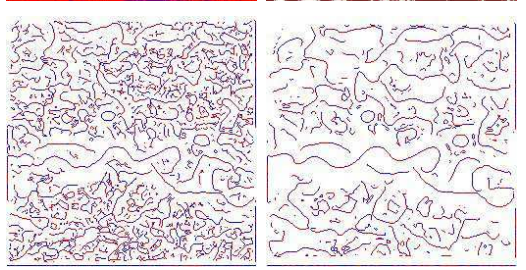

$\mathrm{D}=7.58 \%$

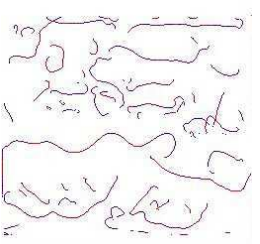

$\mathrm{D}=3.82 \%$

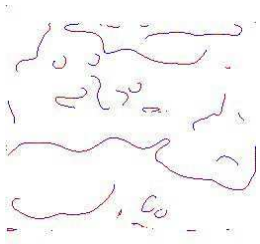

$\mathrm{D}=3.23 \%$

In this context, one might argue about a comparison with Elder-Zucker's algorithm [49]. Elder-Zucker's algorithm on the detection of edges is inspired by its own local scale control method that determines a unique scale, as a function of filter scale $\sigma$, for local estimation at each point in an image [49, 50]. Therefore, a minimum reliable scale i.e., a filter with smallest standard deviation $\sigma$ that can be used reliably, is being determined for each point in the gradient map and used locally to derive logical inferences from derivative estimates of the signal (that are key to edge detection). The choice of $\sigma$ and the definition of reliability rest with the prior computation of a critical value function that depends on some statistical parameters [57]. A good choice of these statistical parameters are necessary for the proper functioning of the algorithm; incorrect estimate may lead to reduced performances. The performance of $\mathbf{I}_{\infty}$, on the other hand, is independent of the choice of any $a$ 
priori inputs and is applied directly on different scale-based representation of images (without doing any processing to enhance the performance of edge detection) and verify its performance across scales. We are interested in extracting the most singular components that are related to edges in an image (by proper computation of singularity exponents on the image, as explained in section 2.2), irrespective of the scale or the spatial representation of the image.

\section{Results}

\subsection{Choice of images}

Images of different entities are chosen trying to cover a broad spectrum of natural images starting from textures (Harrington weave) to an object (car), face (lena) to landscapes (imk01310, imk03324) and signals of turbulent acquisitions (sea surface temperature, turbulence degraded atmospheric phase) as is shown in Table 1 and Table 7 . These experimental images are chosen from standard databases like SIPI image database (Harrington weave, Hibiscus, Lena) [66], CMU image database (car) [67] and the van Hateren database (imk01310, imk03324, imk04050) [27]. The SST data was acquired by MODIS satellite and the perturbated phase data is provided by the french aerospace lab ONERA.

\subsection{Experimental procedure}

We make use of natural images described in section 4.1 for our experiments. The classical edge algorithms tested are Matlab $\left.{ }^{(}\right)$implementations. Reconstruction is performed from edges, calculated using different edge detector algorithms, using equation (19). The pixel densities of all the edges calculated using different edge detectors are kept within a close range (given the constraint imposed by the Gaussian sigma), with the pixel density of $\mathbf{I}_{\infty}$ kept lower than the others. Similar experiment is repeated over the images by adding different proportions of Gaussian white noise with respect to the standard deviation of the input images (see Table 8 and Table 10).

\subsection{Quantitative analysis of the results}

The images we use, together with their reconstruction using different edge detectors, are shown in Table 6 and Table 7. Performance of the reconstruction under different levels of noise $(\mathrm{SNR}=26 \mathrm{~dB}, 14 \mathrm{~dB}$ and $6 \mathrm{~dB})$ is shown in Table 8. Visual quality of the reconstructed images, shown in Table 6, 

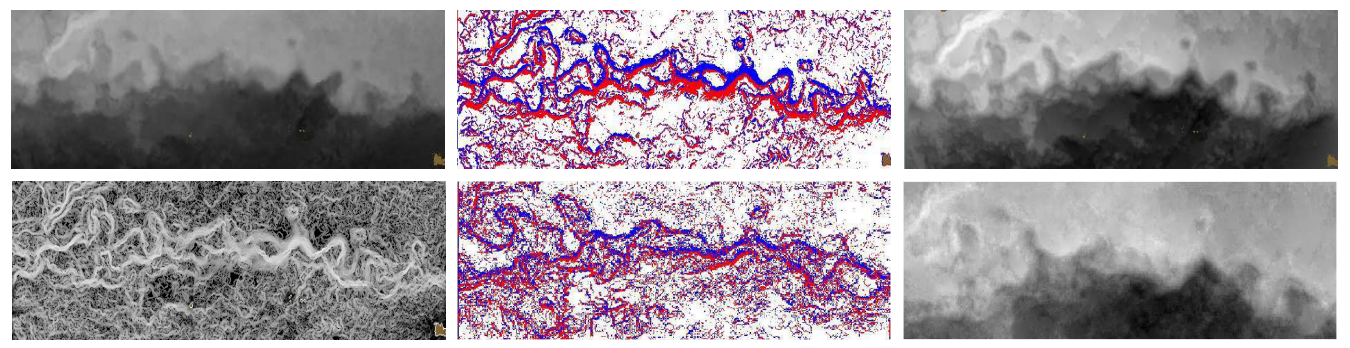

Figure 1: This figure illustrates the complexity of edge detection in the case of turbulent images. Top row (from left to right): excerpt of the Agulhas current from the SST image, set of edge pixels computed by $\mathbf{I}_{\infty}$ corresponding to $25 \%$ of pixel density in the selected area, reconstruction with algorithm $\mathbf{I}_{\infty}$. Bottom row (from left to right): singularity exponents of the SST image, edges produced by algorithm NLFS [53] which behaves the best among the classical edge operators tested, reconstruction with edge pixels produced by NLFS. The coherent structures are not respected by NLFS, showing the superiority of $\mathbf{I}_{\infty}$.

Table 7 and Table 8, are evaluated based on the structural similarity index metric (SSIM) [68]. SSIM's for the reconstructed images shows that $\mathbf{I}_{\infty}$ outperforms the classical edge detectors in majority of the cases.

We also illustrate the pertinence of the framework of reconstructible systems for evaluating an edge operator's compact representation effectiveness using the mean square error (MSE) and peak signal to noise ratio (PSNR, expressed in decibels $\mathrm{dB}$ ) defined by:

$$
\begin{gathered}
\mathrm{MSE}=\frac{1}{m \times n} \sum_{i, j}\left|I\left(x_{i, j}\right)-I_{r}\left(x_{i, j}\right)\right|^{2} \\
\mathrm{PSNR}=20.0 \times \log _{10} \frac{\max (I(\vec{x}))}{\sqrt{\mathrm{MSE}}}
\end{gathered}
$$

Table 9 shows the performance evaluation of the reconstructed images using PSNR and MSE metric and Table 10 shows the performance of the reconstruction under different levels of input SNR using the same metrics.

\subsection{Comments: The case of turbulent signals}

Examination of the results for SST and phase (turbulent phenomena) images are particularly interesting: an edge in a turbulent signal is poorly 
characterized by a filter's response to step functions, and the case of Fully Developped Turbulence is paradigmatic for the existence of a multiscale hierarchy associated to cascading dynamics of physical variables [31]. Tuning with the scale-space parameter given by the kernel's standard deviation modifies the input signal incoherently w.r.t. to the real multiscale hierarchy present in the data, resulting in bad reconstructions for most Gaussian prefiltered edge operators, and with no enhancing by adding more edge pixels. Universality classes are not well characterized by the transitions associated to classical edge operators. Among these operators, NLFS seems to behave the best, yet $\mathbf{I}_{\infty}$ outperforms it, and provides also an automatic threshold determination for the set of edges. In Fig 1, we show an extended part of the SST data and illustrate the sensitivity of edge detection and signal reconstruction between $\mathbf{I}_{\infty}$ and NLFS.

\section{Discussion}

In this article, we presented a performance evaluation of the quality of edge pixels based on the reconstruction formula presented in section 2.3. It happens that $\mathbf{I}_{\infty}$ edge pixels lead, for most images used in this work, the best quantitative results in terms of PSNR, SSIM and MSE. But $\mathbf{I}_{\infty}$ edge pixels are defined irrespective of any reconstruction formula. Consequently, the evaluation procedure based on the reconstruction formula is independent of the definition of edge pixels. In fact, it is easy to see that any reconstruction formula designed to test the accuracy of a compact representation must have, under reasonable hypothesis the form displayed in equation (19). Indeed, if one suppose that a reconstructor is able to generate the whole signal from its set $\mathcal{K}$ of edges, using a linear functional $\mathcal{G}$, then equation (11) must be valid, with $\mathcal{F}_{\infty}$ replaced by $\mathcal{K}$. Then assuming linearity, translational invariance and isotropy, one gets the following reconstruction formula for testing the validity of the set $\mathcal{K}$ of edge pixels:

$$
\widehat{I(\vec{\omega})}=\frac{\langle\vec{\omega} \mid \widehat{\nabla I \mid \mathcal{K}}\rangle}{i\|\vec{\omega}\|^{2}}
$$

In that sense, we can say that the reconstruction technique presented in this work is universal and not specifically designed for the assumptions motivating the article and suitable for the proposed approach. 


\section{Conclusion}

Tested classical edge detection algorithms are reviewed in this work in the sense that they lack compact representation. When considering images as acquisitions of a complex system with unknown extented phase space, edge pixels correspond to high-order transitions, which are characterized by critical exponents. These exponents define universality classes, they describe common statistical and macroscopic multiscale characteristics of the system. When they are numerically evaluated at high precision through wavelet projection measures in a microcanonical setting, they allow the determination of the multiscale hierarchy, of multifractal nature, which is very different to the scale-space models commonly used. The framework of reconstructible system permits a quantitative evaluation of the compactness of a representation, leading to extremely accurate edge pixels, specifically in the case of turbulent data, where classical edge detectors fail. The introduced edge operator $\mathbf{I}_{\infty}$ outperforms on natural images and geophysical variables.

\section{Acknowledgment}

Suman Kumar Maji's PhD is funded by a CORDIS grant and Région Aquitaine OPTAD research project grant.

\section{References}

[1] K. S. Shanmugham, F. M. Dickey, J. A. Green: An Optimal Frequency Domain Filter for Edge Detection in Digital Pictures, IEEE PAMI, 1, 37-49, 1979.

[2] A. Rosenfeld: The Max Roberts Operator is a Hueckel-Type Edge Detector, IEEE PAMI, 3, 101-102, 1981.

[3] S. W. Zucker, R. A. Hummel: A Three-Dimensional Edge Operator, IEEE PAMI, 3, 324-331, 1981.

[4] D. G. Morgenthaler, A. Rosenfeld: Multidimensional Edge Detection by Hypersurface Fitting, IEEE PAMI , 3, 482-486, 1981.

[5] H. K. Liu: Two- and three-dimensional boundary detection, Comput. Graphics Image Processing, 6, 123-134, 1977. 
Table 6: Results of reconstruction over different edge detection algorithms. Image description available in Table 1.
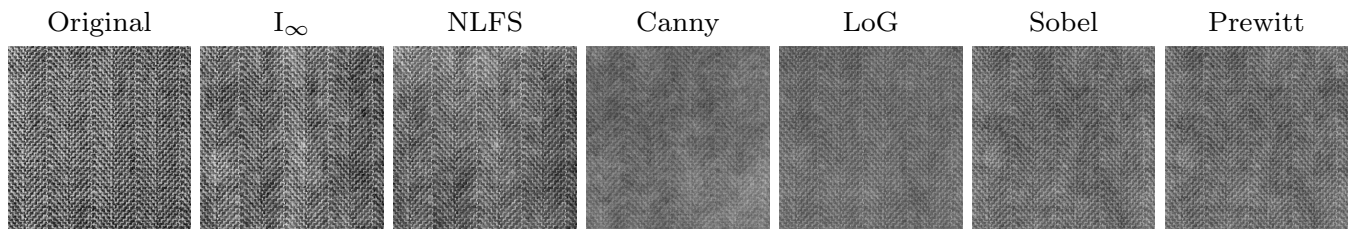

$\operatorname{SSIM}=1$

$\operatorname{SSIM}=0.9881 \quad \mathrm{SSIM}=0.9848$
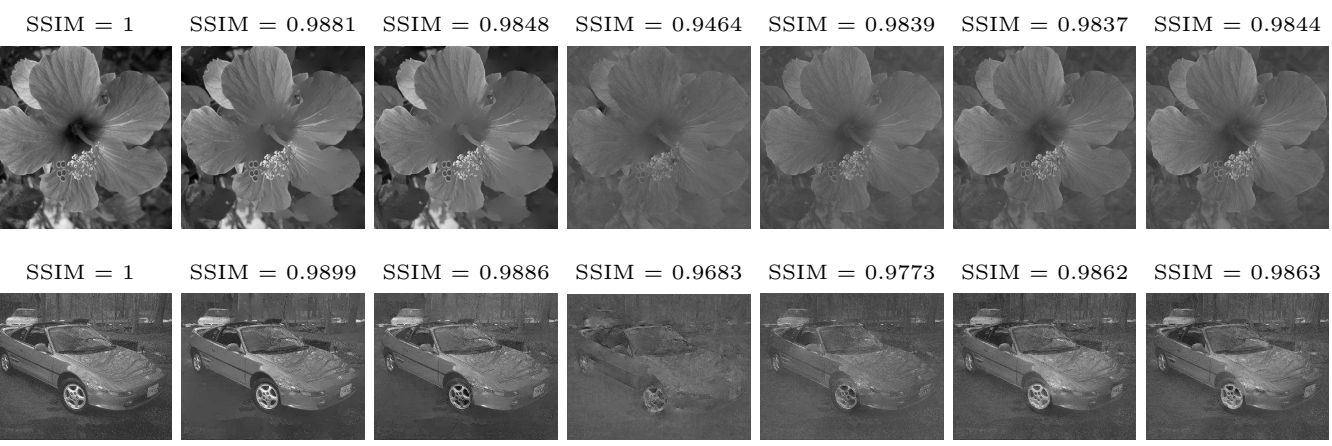

$\operatorname{SSIM}=1$

$\operatorname{SSIM}=0.9921$
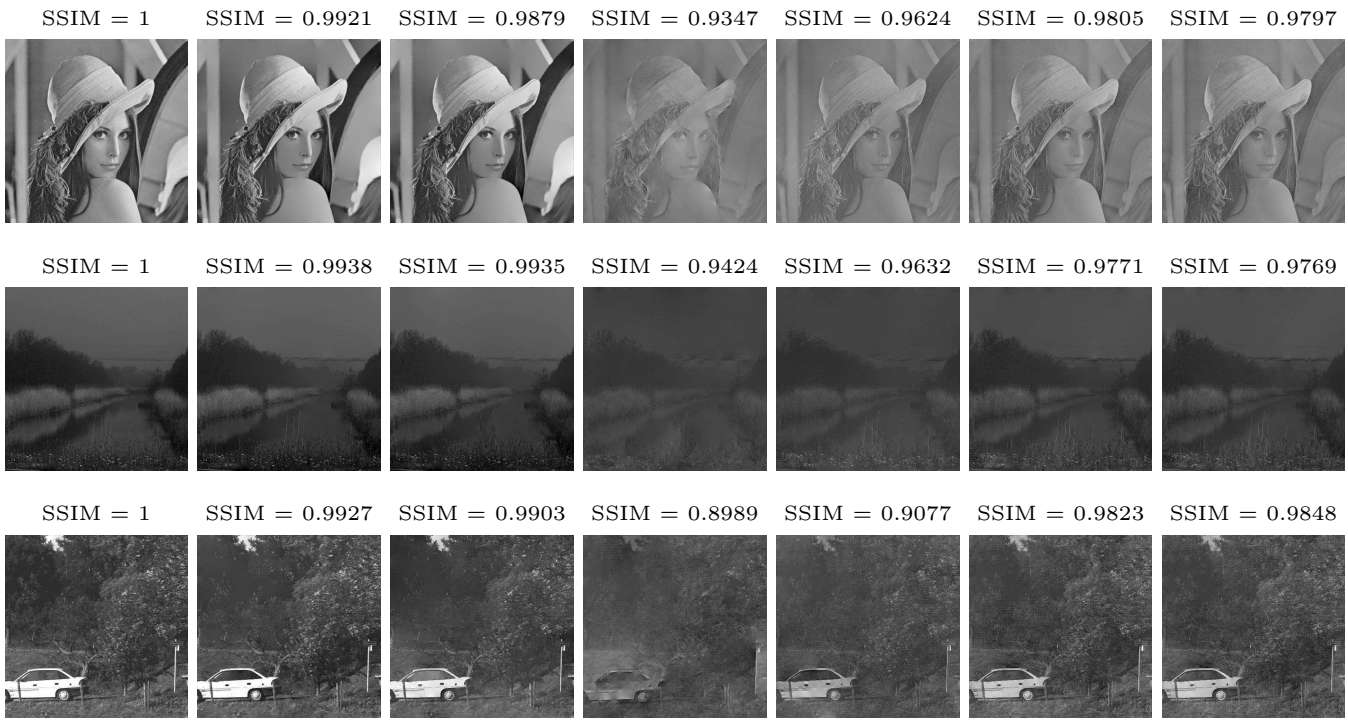

$\mathrm{SSIM}=1$
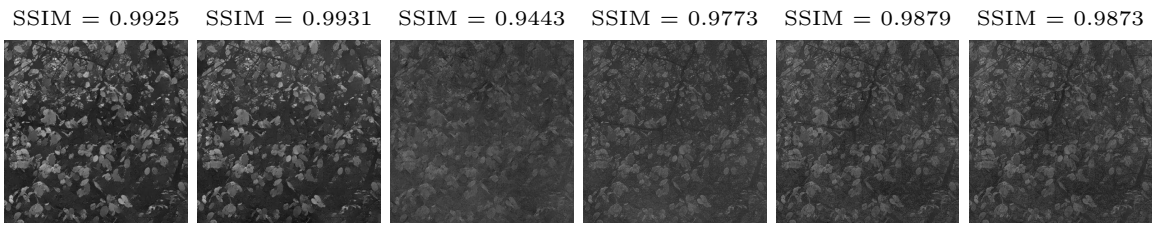

$\mathrm{SSIM}=1$

$\mathrm{SSIM}=0.9954 \quad \mathrm{SSIM}=0.9924$

$\mathrm{SSIM}=0.9621$

SSIM $=0.9784 \quad$ SSIM $=0.9911 \quad$ SSIM $=0.9913$ 
Table 7: Reconstruction results from different edge detection algorithms over turbulent data.

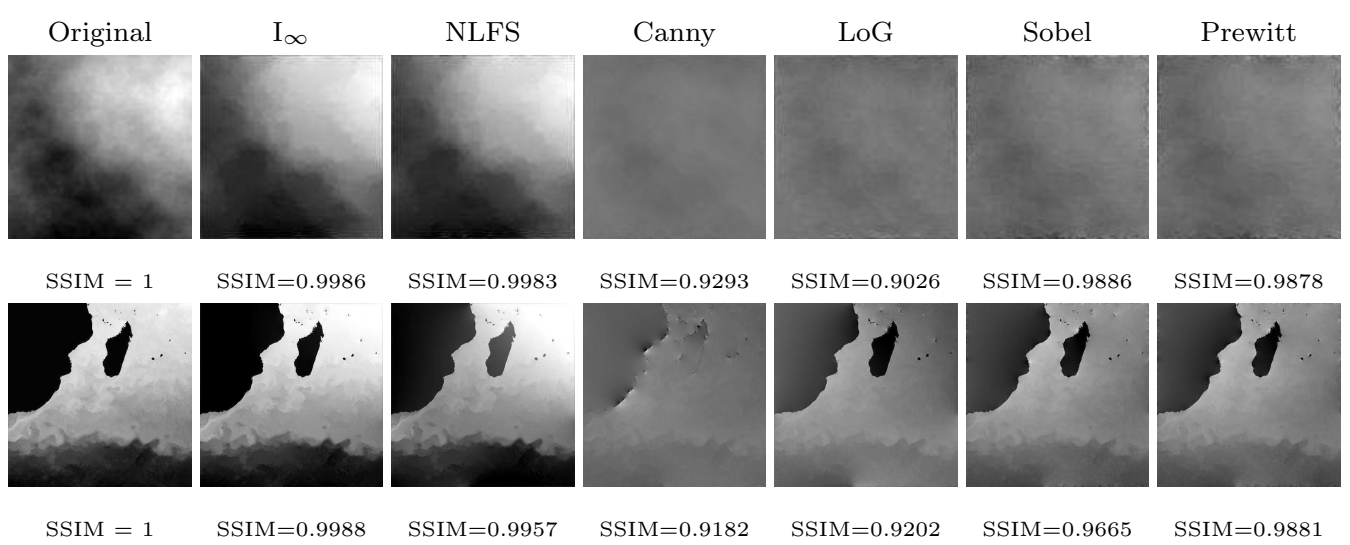

Table 8: Performance under noise: reconstruction results on different edge detection algorithms under different levels of Gaussian white noise.
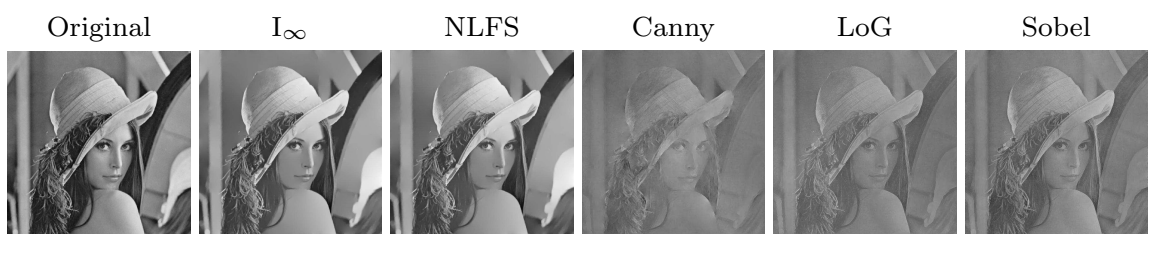

Prewitt

$\mathrm{SNR}=26 \mathrm{~dB} \quad$ psnr $=20.94 \mathrm{~dB} \quad \mathrm{psnr}=20.74 \mathrm{~dB} \quad \mathrm{psnr}=10.75 \mathrm{~dB}$

$\mathrm{psnr}=12.83 \mathrm{~dB}$

psnr $=14.88 \mathrm{~dB}$
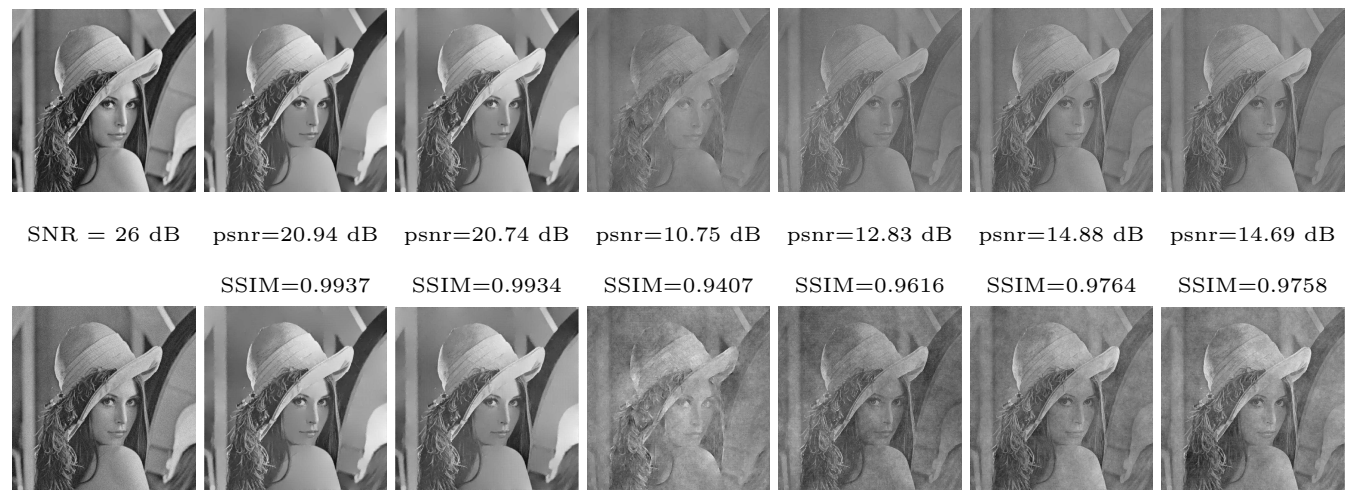

$\mathrm{psnr}=14.69 \mathrm{~dB}$

$\mathrm{SNR}=14 \mathrm{~dB}$

psnr $=18.42 \mathrm{~dB} \quad$ psnr $=18.00 \mathrm{~dB}$

psnr $=12.40 \mathrm{~dB}$

psnr $=13.71 \mathrm{~dB}$

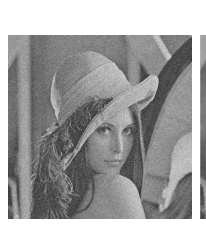

$\mathrm{SSIM}=0.9932$
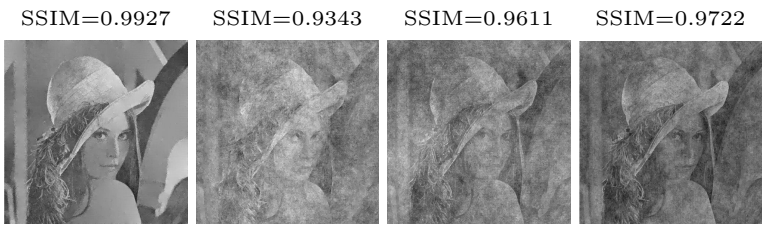

$\mathrm{SNR}=06 \mathrm{~dB}$

$\mathrm{psnr}=15.82 \mathrm{~dB} \quad$ psnr $=15.76 \mathrm{~dB} \quad \mathrm{psnr}=10.02 \mathrm{~dB}$

psnr $=12.18 \mathrm{~dB}$

psnr $=13.26 \mathrm{~dB}$

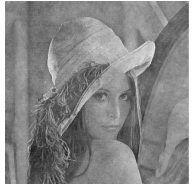

$\operatorname{SSIM}=0.9895$

$\operatorname{SSIM}=0.9886$

SSIM $=0.9206$

$\operatorname{SSIM}=0.9524$

$\operatorname{SSIM}=0.9650$

$\operatorname{SSIM}=0.9755$

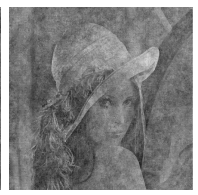

psnr $=13.47 \mathrm{~dB}$

$\mathrm{SSIM}=0.9686$ 
Table 9: Quantitative analysis: evaluation of the reconstruction over different edge detection algorithms, in terms of PSNR and MSE, correspnding to Table 6 and Table 7 . The pixel density (D) of all the edges are kept within a close range, with the pixel density of $\mathbf{I}_{\infty}$ being the lowest.

\begin{tabular}{|c|c|c|c|c|c|c|c|c|}
\hline Image & Params & $\mathrm{I}_{\infty}$ & NLFS & Canny & Lindeberg & LoG & Sobel & Prewitt \\
& & & {$[53]$} & & {$[60]$} & & & \\
\hline weave & D (\%) & 31.5 & 32.87 & 29.56 & 31.86 & 31.28 & 31.57 & 31.67 \\
$(1024 \times 1024$ & mse & 0.1439 & 0.1977 & 0.6600 & 0.3955 & 0.2790 & 0.2148 & 0.2158 \\
pixels) & psnr (dB) & 17.38 & 16.01 & 10.76 & 13.99 & 14.51 & 15.64 & 15.62 \\
\hline hibiscus & D (\%) & 28.55 & 28.87 & 28.55 & 29.15 & 29.98 & 29.43 & 29.22 \\
$(512 \times 512$ & mse & 0.0906 & 0.0944 & 0.4005 & 0.3139 & 0.2657 & 0.1690 & 0.1622 \\
pixels) & psnr (dB) & 22.43 & 21.92 & 16.04 & 17.04 & 17.76 & 19.72 & 19.80 \\
\hline car & D (\%) & 30.00 & 36.03 & 32.28 & 36.28 & 37.58 & 36.86 & 37.24 \\
$(260 \times 320$ & mse & 0.0794 & 0.1321 & 0.7350 & 0.3972 & 0.4038 & 0.2150 & 0.2262 \\
pixels) & psnr (dB) & 25.59 & 23.37 & 15.84 & 18.68 & 18.53 & 21.26 & 21.04 \\
\hline Lena & D (\%) & 32.00 & 32.93 & 28.80 & 32.20 & 32.36 & 30.13 & 30.26 \\
$(512 \times 512$ & mse & 0.0563 & 0.0587 & 0.5898 & 0.3718 & 0.3775 & 0.2298 & 0.2305 \\
pixels) & psnr (dB) & 21.17 & 20.99 & 10.98 & 12.98 & 12.91 & 15.06 & 15.05 \\
\hline imk01310 & D (\%) & 30 & 30.12 & 27.98 & 31.09 & 31.24 & 29.90 & 30.40 \\
$(512 \times 512$ & mse & 0.0626 & 0.0923 & 1.1389 & 1.0744 & 0.9434 & 0.2150 & 0.1933 \\
pixels) & psnr (dB) & 29.43 & 27.74 & 16.88 & 17.08 & 17.65 & 24.07 & 24.53 \\
\hline imk03324 & D (\%) & 30.00 & 30.14 & 32.48 & 35.43 & 36.46 & 36.06 & 36.82 \\
$(512 \times 512$ & mse & 0.0854 & 0.1057 & 0.7808 & 0.3879 & 0.3168 & 0.1634 & 0.1705 \\
pixels) & psnr (dB) & 23.08 & 22.15 & 13.42 & 16.51 & 17.38 & 20.26 & 20.07 \\
\hline imk04050 & D (\%) & 31.00 & 31.32 & 31.22 & 32.59 & 32.95 & 32.61 & 32.93 \\
$(512 \times 512$ & mse & 0.0652 & 0.0974 & 0.4634 & 0.3554 & 0.2996 & 0.1358 & 0.1361 \\
pixels) & psnr (dB) & 25.79 & 24.05 & 17.23 & 19.17 & 22.60 & 20.50 & 22.59 \\
\hline phase & D (\%) & 25.00 & 27.27 & 24.63 & 23.62 & 29.15 & 25.91 & 25.42 \\
$(128 \times 128$ & mse & 0.0184 & 0.0187 & 0.8025 & 1.3023 & 0.9307 & 0.1660 & 0.1745 \\
pixels) & psnr (dB) & 23.97 & 23.14 & 7.08 & 5.29 & 5.34 & 14.24 & 14.02 \\
\hline SST & D (\%) & 25.00 & 30.73 & 21.62 & 22.64 & 23.95 & 23.80 & 24.01 \\
$(512 \times 512$ & mse & 0.0114 & 0.0404 & 0.8105 & 0.8257 & 0.7545 & 0.2035 & 0.2011 \\
pixels) & psnr (dB) & 23.99 & 18.50 & 5.56 & 5.40 & 5.79 & 11.48 & 11.54 \\
\hline
\end{tabular}

[6] W. H. H. J. Lunscher: The Asymptotic Optimal Frequency Domain Filter for Edge Detection, IEEE PAMI, 5, 678-680, 1983.

[7] W. H. H. J. Lunscher, M. P. Beddoes: Optimal Edge Detector Design I: Parameter Selection and Noise Effects, IEEE PAMI, 8, 164-177, 1986.

[8] W. H. H. J. Lunscher, M. P. Beddoes: Optimal Edge Detector Design II: Coefficient Quantization, IEEE PAMI, 8, 178-187, 1986. 
Table 10: Quantitative analysis (noisy environment): evaluation of the reconstruction over different edge detection algorithms, in terms of PSNR and MSE, under different levels of noise. For all images, the first, second and third row represents the performance under input SNR of $26 \mathrm{~dB}, 14 \mathrm{~dB}$ and $6 \mathrm{~dB}$ respectively.

\begin{tabular}{|c|c|c|c|c|c|c|c|}
\hline Image & $\begin{array}{c}\mathrm{I}_{\infty} \\
\text { psnr , mse }\end{array}$ & $\begin{array}{c}\text { NLFS } \\
\text { psnr, mse }\end{array}$ & $\begin{array}{l}\text { Canny } \\
\text { psnr, mse }\end{array}$ & $\begin{array}{c}\text { Lindeberg } \\
\text { psnr, mse }\end{array}$ & $\begin{array}{c}\text { LoG } \\
\text { psnr , mse }\end{array}$ & $\begin{array}{c}\text { Sobel } \\
\text { psnr, mse }\end{array}$ & $\begin{array}{l}\text { Prewitt } \\
\text { psnr, mse }\end{array}$ \\
\hline weave & $\mid \begin{array}{ll}16.89, & 0.1763 \\
16.68, & 0.2388 \\
14.51, & 0.5388\end{array}$ & $\begin{array}{l}15.49,0.2384 \\
15.21,0.3301 \\
14.61,0.6217\end{array}$ & $\begin{array}{cc}10.37, & 0.7858 \\
10.05, & 0.8449 \\
9.75, & 1.0993\end{array}$ & $\begin{array}{l}13.34,0.4176 \\
13.12,0.4986 \\
12.78,0.8647\end{array}$ & $\begin{array}{l}14.45,0.2964 \\
14.24,0.4103 \\
13.53,0.7520\end{array}$ & $\begin{array}{l}15.60,0.2278 \\
15.48,0.3344 \\
13.98,0.6500\end{array}$ & \begin{tabular}{|ll}
15.51, & 0.2356 \\
15.22, & 0.3245 \\
13.57, & 0.6169 \\
\end{tabular} \\
\hline hibiscus & $\left\{\begin{array}{l}22.18,0.0954 \\
20.64,0.1553 \\
17.66,0.4300\end{array}\right.$ & $\begin{array}{l}21.88,0.1078 \\
20.24,0.1708 \\
16.07,0.4420 \\
\end{array}$ & $\begin{array}{ll}16.01, & 0.4336 \\
15.26, & 0.5146 \\
13.49, & 0.9930 \\
\end{array}$ & \begin{tabular}{ll|}
16.76, & 0.3277 \\
16.11, & 0.4350 \\
14.81, & 0.6040
\end{tabular} & $\begin{array}{l}17.72,0.2755 \\
16.93,0.3385 \\
14.20,0.8359 \\
\end{array}$ & $\begin{array}{ll}19.69, & 0.1762 \\
18.96, & 0.2590 \\
15.53, & 0.6033\end{array}$ & \begin{tabular}{|ll}
19.66, & 0.1728 \\
18.29, & 0.2685 \\
16.24, & 0.6487 \\
\end{tabular} \\
\hline car & $\begin{array}{l}25.48,0.0837 \\
23.69,0.1416 \\
19.41,0.3952\end{array}$ & $\begin{array}{l}22.87,0.1535 \\
22.05,0.2189 \\
17.96,0.4794 \\
\end{array}$ & $\begin{array}{l}15.80,0.7351 \\
15.72,0.7951 \\
15.40,1.0349 \\
\end{array}$ & \begin{tabular}{ll|}
18.57, & 0.4000 \\
18.11, & 0.4668 \\
17.67, & 0.5550
\end{tabular} & $\begin{array}{ll}18.52, & 0.4040 \\
18.12, & 0.4612 \\
16.61, & 0.7574 \\
\end{array}$ & \begin{tabular}{ll|}
21.22, & 0.2260 \\
20.81, & 0.2796 \\
18.39, & 0.9708 \\
\end{tabular} & $\begin{array}{|ll|}20.99, & 0.2364 \\
20.02, & 0.3071 \\
17.98, & 0.5778 \\
\end{array}$ \\
\hline imk01310 & $\begin{array}{l}29.23,0.0627 \\
26.21,0.1247 \\
19.64,0.3872\end{array}$ & $\begin{array}{l}27.53,0.0951 \\
25.23,0.1595 \\
18.75,0.4196 \\
\end{array}$ & $\begin{array}{l}16.82,1.1749 \\
15.98,1.3719 \\
13.28,1.5785 \\
\end{array}$ & $\begin{array}{l}16.96,1.0853 \\
16.18,1.1278 \\
15.33,1.2092\end{array}$ & $\begin{array}{l}17.57,0.9494 \\
17.45,0.9709 \\
14.23,1.3095 \\
\end{array}$ & $\begin{array}{l}23.83,0.2232 \\
22.34,0.3155 \\
16.67,0.6838\end{array}$ & \begin{tabular}{|ll}
24.46, & 0.1938 \\
22.16, & 0.2756 \\
17.99, & 0.6100 \\
\end{tabular} \\
\hline imk03324 & $\mid \begin{array}{ll}23.07, & 0.0921 \\
22.16, & 0.1551 \\
18.82, & 0.4310\end{array}$ & $\begin{array}{ll}21.98, & 0.1216 \\
21.80, & 0.1568 \\
18.22, & 0.4578 \\
\end{array}$ & $\begin{array}{ll}13.39, & 0.8802 \\
13.13, & 0.8853 \\
12.10, & 0.9979 \\
\end{array}$ & $\begin{array}{ll}16.32, & 0.3941 \\
16.08, & 1.1278 \\
15.80, & 0.5712 \\
\end{array}$ & $\begin{array}{l}17.34,0.3571 \\
17.15,0.4636 \\
16.13,0.6700 \\
\end{array}$ & $\begin{array}{ll}20.10, & 0.1932 \\
19.52, & 0.2753 \\
18.13, & 0.5482 \\
\end{array}$ & \begin{tabular}{|ll}
19.43, & 0.2217 \\
19.33, & 0.2911 \\
17.40, & 0.5882 \\
\end{tabular} \\
\hline imk04050 & $\mid \begin{array}{ll}25.53, & 0.0696 \\
23.25, & 0.1317 \\
18.06, & 0.4295\end{array}$ & $\begin{array}{l}23.95,0.1005 \\
21.80,0.1697 \\
17.66,0.4450 \\
\end{array}$ & $\begin{array}{ll}17.21, & 0.4712 \\
16.46, & 0.5960 \\
14.35, & 0.9353 \\
\end{array}$ & $\begin{array}{l}18.35,0.3591 \\
17.00,0.4874 \\
14.89,0.8137\end{array}$ & $\begin{array}{l}18.95,0.3149 \\
18.05,0.3875 \\
15.36,0.7492 \\
\end{array}$ & $\begin{array}{ll}22.49, & 0.1429 \\
20.60, & 0.2379 \\
16.59, & 0.6069 \\
\end{array}$ & \begin{tabular}{|ll}
22.45, & 0.1422 \\
19.74, & 0.2580 \\
16.07, & 0.6592 \\
\end{tabular} \\
\hline phase & $\left\{\begin{array}{l}23.30,0.0230 \\
19.02,0.0883 \\
15.39,0.3784\end{array}\right.$ & $\begin{array}{l}22.77,0.0261 \\
19.35,0.0884 \\
15.87,0.3693 \\
\end{array}$ & $\begin{array}{l}7.69,0.8889 \\
7.17,0.9437 \\
6.59,0.9051 \\
\end{array}$ & $\begin{array}{l}5.15,1.4985 \\
5.01,1.6293 \\
4.59,2.2028 \\
\end{array}$ & $\begin{array}{l}6.55,1.0722 \\
6.00,1.2843 \\
5.03,1.3440 \\
\end{array}$ & $\begin{array}{c}13.07,0.2603 \\
10.65,0.5678 \\
9.80,1.1675 \\
\end{array}$ & \begin{tabular}{|cc}
12.63, & 0.2604 \\
10.97, & 0.5775 \\
9.26, & 0.9469
\end{tabular} \\
\hline SST & $\begin{array}{l}23.47,0.0159 \\
19.21,0.0822 \\
15.09,0.3686\end{array}$ & $\begin{array}{l}18.02,0.0427 \\
17.85,0.0979 \\
15.03,0.3797\end{array}$ & $\begin{array}{l}5.22,0.8383 \\
5.09,0.9554 \\
4.86,1.3826\end{array}$ & $\begin{array}{l}5.06,0.8354 \\
4.88,0.8536 \\
4.10,0.9447\end{array}$ & $\begin{array}{l}5.41,0.7707 \\
4.96,0.8102 \\
4.14,0.9676\end{array}$ & $\begin{array}{c}10.11,0.3280 \\
9.74,0.4179 \\
9.00,0.7962\end{array}$ & $\mid \begin{array}{cc}10.74, & 0.2852 \\
10.53, & 0.3253 \\
9.76, & 0.7958\end{array}$ \\
\hline
\end{tabular}

[9] M. E. Jernigan, R. W. Wardell: Does the eye contain optimal edge detection mechanisms, IEEE Trans. Syst., Man, Cybern., SMC-11, 441-444, 1981.

[10] D. Marr, E. Hildreth: Theory of edge detection, Proc. Royal Soc. London $B, 207,187-217,1980$.

[11] R. M. Haralick: Digital Step Edges from Zero Crossing of Second Directional Derivatives, IEEE PAMI, 6, 58-68, 1984.

[12] A. J. Tabatabai, R. Mitchell: Edge Location to Subpixel Values in Digital Imagery, IEEE PAMI, 6, 188-201, 1984. 
[13] I. Pitas, A. N. Venetsalopoulous: Edge Detectors Based on Nonlinear Filters, IEEE PAMI, 8, 538-550, 1986.

[14] J. Canny: A Computational Approach to Edge Detection, IEEE PAMI, 8, 679-698, 1986.

[15] V. S. Nalwa, T. O. Binford: On Detecting Edges, IEEE PAMI, 8, 699$714,1986$.

[16] V. Torre, T. A. Poggio: On edge detection, IEEE PAMI, 8, 147-163, 1986.

[17] E. Hildreth: Implementation of a theory of edge detection, Massachusetts Inst. Technol., Cambridge, A. 1. Memo 579, 1980.

[18] O. Faugeras: Three-dimensional computer vision:a geometric viewpoint, MIT Press, 1993, ISBN: 0-262-06158-9.

[19] A. L. Yuille, T. A. Poggio: Scaling Theorems for Zero Crossings, IEEE PAMI, 8, 15-25, 1986.

[20] A. J. Bell, T. J. Sejnowski: The independent components of natural scenes are edge filters, Vision Research, 37, 3327-3338, 1997.

[21] M. C. Morrone, D. C. Burr: Feature detection in human vision: A phasedependent energy model, Proceedings of the Royal Society of London. Series B, biological sciences, 1988.

[22] P. Kovesi: Image features from phase congruency, Journal of Computer Vision Research, 1999.

[23] Z. Wang, E. P. Simoncelli: Local phase coherence and the perception of blur, Advances in Neural Information Processing Systems, vol. 16, 2004.

[24] A. Turiel, N. Parga: The Multifractal Structure of Contrast Changes in Natural Images: From Sharp Edges to Textures, Neural Computation, 12, 763-793, 2000.

[25] J. J. Atick: Could information theory provide an ecological theory of sensory processing, Network: Comput. Neural Syst., 3, 213-251, 1992. 
[26] J. H. van Hateren: Theoretical predictions of spatiotemporal receptive fields of fly LMCS, and experimental validation, J. Comp. Physiology A, 171, 157-170, 1992.

[27] J. H. van Hateren, A. van der Schaaf: Independent component filters of natural images compared with simple cells in primary visual cortex, Proc.R. Soc. Lond., B265, 359-366, 1998.

[28] A. Lesne: Méthodes de renormalisation, Eyrolles Science, 1995, ISBN: 2-212-05830-6.

[29] A. Witkin: Scale-space filtering, Proc. IJCAI, Karlsruhe, West Germany, 1019-1021, 1983.

[30] T. Lindeberg: Scale-Space Theory in Computer Vision, Kluwer Academic Publishers, 1994, ISBN: 0-7923-9418-6.

[31] U. Frisch: Turbulence, Cambridge Univ. Press, 1995, Cambridge MA.

[32] F. Bergholm: Edge Focusing, IEEE PAMI, 9, 726-741, 1987.

[33] J. J. Clark: Singularity Theory and Phantom Edges in Scale Space, IEEE PAMI, 10, 720-727, 1988.

[34] P. Bouthemy: A Maximum Likelihood Framework for DeterminingMoving Edges, IEEE PAMI, 11, 499-511, 1989.

[35] P. Perona, J. Malik: Scale-Space and Edge Detection Using Anisotropic Diffusion, IEEE PAMI, 12, 629-639, 1990.

[36] P. Sarkar, K. L. Boyer: On Optimal Infinite Impulse Response Edge Detection Filters, IEEE PAMI, 13, 1154-1171, 1991.

[37] P. Jeong, C. I. Kim: Adaptative Determination of Filter Scales for Edge Detection, IEEE PAMI, 14, 579-585, 1992.

[38] S. Mallat, S. Zhong: Characterization of Signals from Multiscale Edges, IEEE PAMI, 14, 710-732, 1992.

[39] A. Arenodo, F. Argoul, E. Bacry, J. Elezgaray, J. Muzy: Ondelettes, Multifractales et Turbulence, Diderot Editeur, Paris, 1995. 
[40] A. Arenodo, E. Bacry, J. Muzy: The thermodynamics of fractals revisited with wavelets, Physica A , 213, 232-275, 1995.

[41] A. Turiel, C. J. Pérz-Vicente, J. Grazzini: Numerical methods for the estimation of multifractal singularity spectra on sampled data: A comparative study, Journal of Computational Physics, 216, 362-390, 2006.

[42] H. Yahia, J. Sudre, C. Pottier, V. Garon: Motion analysis in oceanographic satellite images using multiscale methods and the energy cascade. Pattern Recognition, 43(10): 3591-3604, 2010.

[43] F. van der Heijden: Edge and Line Feature Extraction Based on Covariance Models, IEEE PAMI, 17, 16-33, 1995.

[44] N. Ahuja: A Transform for Multiscale Image Segmentation by Integrated Edge and Region Detection, IEEE PAMI, 18, 1211-1235, 1996.

[45] S. Geman, D. Geman: Stochastic Relaxation, Gibbs Distribution and the Bayesian Restoration of Images, IEEE PAMI, 6, 721-741, 1984.

[46] P. Liang, Y. W. Wang: Local Scaled Controlled Anisotropic Diffusion with Local Noise Estimate for Image Smoothing and Edge Detection, ICCV, Narosa Publishing House, 193-200, 1998.

[47] D. H. Marimont, Y. Rubner: A Probabilistic Framework for Edge Detection and Scale Selection, ICCV, Narosa Publishing House, 207-214, 1998.

[48] S. Z. Li: Close-Form Solution and Parameter Selection for Convex Minimization-Based Edge-Preserving Smoothing, IEEE PAMI, 20, 916932, 1998.

[49] J. H. Elder, S. W. Zucker: Local Scale Control for Edge Detection and Blur Estimation, IEEE PAMI, 20, 699-716, 1998.

[50] J. H. Elder, S. W. Zucker: Local scale control for edge detection and blur estimation, In Proc. 4th European Conf. on Computer Vision, 1996b, Lecture Notes in Computer Science, Springer Verlag, New York, pp. 5769.

[51] K. Suzuki, I. Oriba, N. Sugie: Neural Edge Enhancer for Supervised Edge Enhancement from Noisy Images, IEEE PAMI, 25, 1582-1596, 2003. 
[52] X. Wang: Laplacian Operator-Based Edge Detectors, IEEE PAMI, 29, 886-890, 2007.

[53] O. Laligant, F. Truchetet: A Nonlinear Derivative Scheme Applied to Edge Detection, IEEE PAMI, 32, 242-257, 2010.

[54] D. Galun, M. Basri, A. Brandt: Multiscale Edge Detection and Fiber Enhancement Using Differences of Oriented Means, ICCV, 2007.

[55] W. Mc Ilhagga: The Canny Edge Detector Revisited, Int. J. Comput. Vis., 91, 251-261, DOI 10.1007/s11263-010-0392-0, 2011.

[56] R. Kimmel, A. M. Bruckstein: Regularized Laplacian Zero Crossings as Optimal Edge Integrators, Int. J. Comput. Vis., 53, 225-243, 2003.

[57] J. H. Elder: Are Edges Incomplete ?, Int. J. Comput. Vis., 34, 97-122, 1999.

[58] S. Mallat: A wavelet tour of signal processing: the sparse way, Academic Press, 3rd edition, 2008.

[59] G. Bofetta, M. Cencini, M. Falcioni, A. Vulpiani: Predictability: a way to characterize Complexity, Physics Reports 356, 367-474, 2001.

[60] T. Lindeberg: Edge detection and ridge detection with automatic scale selection, International Journal of Computer Vision, 30 ,117-154, 1998.

[61] T. Lindeberg, ter Haar Romeny: Linear scale-space: (I) Basic theory and (II) Early visual operations, Geometry-Driven Diffusion, pp. 1-77, Kluwer Academic Publishers/Springer, Dordrecht, Netherlands, 1994.

[62] H. E. Stanley: Introduction to Phase Transitions and Critical Phenomena, Oxford Science Publications, ISBN: 0-19-505316-8, 1971.

[63] A. Turiel, A. del. Pozo: Reconstructing images from their most singular fractal manifold, IEEE Transactions Image Processing, 2002, 11, 345-50.

[64] A. Agrawal, R. Raskar, R. Chellappa: What is the Range of Surface Reconstructions from a Gradient Field?, ECCV, 2006.

[65] D. J. Fried: Relations between the statistics of natural images and the response properties of cortical cells, J. Opt. Soc. Amer., vol. 4, pp. 23792394, 1987. 
[66] USC-SIPI image database. http://sipi.usc.edu/services/database (1981).

[67] Carnegie Mellon image database. http://vasc.ri.cmu.edu/idb/.

[68] Z. Wang, A. C. Bovik, H. R. Sheikh, E. P. Simoncelli, Image quality assessment: From error visibility to structural similarity, IEEE Transactios on Image Processing, vol. 13, no. 4, pp. 600-612, Apr. 2004.

[69] O. Pont, A. Turiel, C. J. Pérez-Vicente: Empirical evidences of a common multifractal signature in economic, biological and physical systems, Physica A, 388, 2025-2035, 2009.

[70] H. Wendt, P. Abry: Multifractality Tests Using Bootstrapped Wavelet Leaders, IEEE Transactions on signal processing, 55, 10, 2007.

[71] A. Turiel, H. Yahia, C. J. Pérez-Vicente: Microcanonical multifractal formalism -a geometrical approach to multifractal systems: Part I. Singularity analysis, J. Phys. A: Math. Theor., 41, 015501, 2008.

[72] O. Pont, A. Turiel, H. Yahia: An optimized algorithm for the evaluation of local singularity exponents in digital signals, IWCIA 2011, 346-357. 